\title{
Visual dysfunction predicts cognitive impairment and white matter degeneration in Parkinson's disease
}

\author{
Angeliki Zarkali ${ }^{1}$, Peter McColgan ${ }^{2}$, Louise-Ann Leyland ${ }^{1}$, Andrew J. Lees ${ }^{3}$, Rimona S. Weil ${ }^{1,4,5}$
}

1. Dementia Research Centre, University College London, 8-11 Queen Square, London, WC1N 3AR

2. Huntington's Disease Centre, University College London, Russell Square House, London, WC1B 5EH

3. Reta Lila Weston Institute of Neurological Studies, 1 Wakefield Street, London, WC1N 1PJ

4. Wellcome Centre for Human Neuroimaging, University College London, 12 Queen Square, London, WC1N 3AR

5. Movement Disorders Consortium, National Hospital for Neurology and Neurosurgery, Queen Square, London, WC1N 3AR 


\section{Introduction}

The risk of dementia is markedly increased in Parkinson's disease (PD) but its onset and severity is highly heterogeneous ${ }^{1,2}$, making individual-level predictions difficult and limiting timely therapeutic interventions. There is now a growing body of evidence that Parkinson's patients with visual dysfunction are at a greater risk of dementia ${ }^{3-5}$, but whether this translates into structural change over time is not yet known.

Animal and cell models suggest that axonal injury is an early event in the degenerative processes leading to Parkinson's dementia ${ }^{6-8}$. In patients with PD, white matter changes are seen and can be measured using diffusion-weighted imaging. These increase with worsening cognition and may precede grey matter atrophy. ${ }^{9,10}$ However, white matter imaging using conventional diffusion-tensor imaging metrics is insensitive and cannot accurately model crossing fibres, which affects a large proportion of white matter tracts. ${ }^{11}$

Recently, higher-order diffusion models, such as Fixel-based analysis (FBA) have emerged as a more sensitive and fibre-specific framework for identifying white matter alterations. ${ }^{12}$ FBA quantifies changes in specific fibre populations within a voxel known as a "fixel", thus allowing comparisons of specific tracts rather than metrics averaged across voxels. ${ }^{12}$ We have recently shown that FBA is more sensitive than standard techniques in identifying white matter changes in patients with $\mathrm{PD}^{13}$. A recent FBA study showed macro-structural changes within the corpus callosum in patients with Parkinson's disease which worsened with disease progression. but there were only limited cognitive assessments and it was not designed to examine white matter changes that relate to cognitive progression in $\mathrm{PD}^{14}$.

We have examined the white matter changes that evolve during the early stages of cognitive impairment in PD. We assessed patients with PD and visual dysfunction, who are known to be at risk of incipient dementia ${ }^{1,15,16}$ and examined white matter alterations at baseline and after 18 months follow-up, comparing them to PD patients with intact visual function. We also examined white matter changes linked with PD and mild cognitive impairment (PD-MCI) in the same group of patients. We assessed white matter changes in patients with PD-MCI at baseline, and longitudinally in PD-MCI and in patients who progressed to develop PD-MCI. We hypothesised that patients with Parkinson's and visual dysfunction would show worsening cognitive performance and diffuse white matter degeneration at follow up, compared to those with intact vision; and that more diffuse changes would be evident in this group than in patients with PD-MCI and PD-MCI converters, reflecting the greater sensitivity of poor vision as a predictor of degeneration in PD. 


\section{Methods}

An overview of the study methodology is seen in Figure 1.

A total of 102 participants were included in the study: 76 patients with PD and 25 unaffected controls. Participants underwent clinical and psychological assessments as well as brain imaging at baseline and again after 18 months (Visit 2). Participants with PD were further classified according to their performance in two computer-based visual tasks, the Cats and Dogs task and Biological Motion task. Details of stimulus generation and experimental procedures have been previously described $^{5,16-18}$. Performance in these tasks have been shown to correlate with cognitive but not lowlevel visual performance at baseline as well as worsening cognition at one-year follow up ${ }^{5,17}$. We have also shown that task performance is associated with fibre-specific white matter changes ${ }^{13}$. Similar to previous work, participants who performed worse than the group median performance on both tasks at baseline were classified as PD low visual performers $(n=22)$. All other participants were classified as PD high visual performers $(\mathrm{n}=54)$.

All participants underwent a series of clinical and psychological assessments at both study time points. All PD participants had a MoCA score $\geq 26$ at baseline, above the cut-off for Parkinson's dementia of the Movement Disorder Society Task Force Criteria ${ }^{19}$. PD with Mild Cognitive Impairment (PD-MCI) was defined as impaired performance ( $<1.5 \mathrm{SD}$ of control performance) on at least two domain neuropsychological tests according to MDS criteria ${ }^{20}$. We examined both PD participants with MCI at baseline ( $\mathrm{n}=13$, of whom 9 were low visual performers) and those who had normal baseline cognition but developed MCI at Visit 2 ( $n=13$ of whom 4 were low visual performers), defined here as PD-MCI ( $n=26)$, in order to capture the group at risk of declining cognitive function. (We also performed sub-analyses of each of these groups separately, to test whether established PD-MCI, or very early changes in the converters might be driving any changes we found). All other PD participants were classified as PD with normal cognition (PD-NC, n=50). A composite cognitive $\mathrm{z}$ score (averaged across the 5 individual cognitive domains) was also computed $^{21}$.

We performed whole brain fixel based analysis using baseline age, gender and total intracranial volume as covariates, whole brain, paired comparisons of fixel-derived metrics were performed at baseline between 1) patients with PD and controls; 2) PD low versus PD high visual performers, and 3) PD-MCI versus PD-NC. Additional comparisons of interest included correlation with MoCA and two clinically-derived dementia risk scores. ${ }^{22,23}$ Whole brain fixel-based analysis refers to the 
comparison of all white matter fixels within the brain using the John Hopkins University (JHU) atlas, as is standard for this analysis. ${ }^{14,24}$

Additionally, to investigate longitudinal change in selective fibre pathways within the visual system, we also performed tract of interest analyses. We selected 11 white matter tracts involved in visual processing, as in our previous work ${ }^{13}$ : Posterior thalamic and optic radiations, Splenium, Body and Genu of the corpus callosum, Superior longitudinal fasciculi, Inferior fronto-occipital fasciculi (segmentation includes the inferior longitudinal fasciculus), and Superior fronto-occipital fasciculi. We then calculated the mean FC across each tract per participant. FC was chosen for tract of interest analysis as prior works have shown it is the most sensitive fibre-specific metric of white matter degeneration in $\mathrm{PD}^{13,14}$. Mean FC at baseline and longitudinally was compared across PD low versus PD high visual performers. Statistical comparison was performed with a linear mixed model (using age, gender and intracranial volume as covariates). A false discovery rate (FDR) correction was performed for the 11 tracts tested, using the Benjamini/Hochberg method. Correlational analyses of mean tract FC with a combined cognitive score was performed using linear regression with age, gender and total intracranial volume as covariates (significance threshold $\mathrm{p}<0.05$ ).

\section{Figure 1. Overview of study methodology (opposite)}

Individual raw diffusion-weighted imaging scans (DWI) underwent preprocessing and constrained spherical deconvolution (CSD) leading to estimation for distribution of fiber orientation (FOD) and calculation of the three fixel metrics for each participant. A study-specific template was created at baseline from 30 randomly selected participants (20 Parkinson's disease and 10 controls). Fixels from each subject were then registered to the template fixels. Whole brain probabilistic tractography with 10 million streamlines was performed on the template image and spherical-deconvolution informed filtering of tractograms was then performed resulting to 2 million streamlines.

Connectivity-based Fixel Enhancement was performed on the resulting streamlines for statistical comparison with 5000 permutations and family-wise error correction across all white matter fixels using the JHU atlas as a mask.

DWI: diffusion-weighted imaging; CSD: multi-shell multi-tissue constrained spherical deconvolution; FOD:fiber orientation distribution; FD: fiber density; FC: fiber-bundle crosssection; FDC: fiber density and cross-section; JHU: Johns Hopkins University. 
bioRxiv preprint doi: https://doi.org/10.1101/2020.10.12.335547; this version posted October 12, 2020. The copyright holder for this

preprint (which was not certified by peer review) is the author/funder, who has granted bioRxiv a license to display the preprint in perpetuity. It is made available under aCC-BY 4.0 International license.

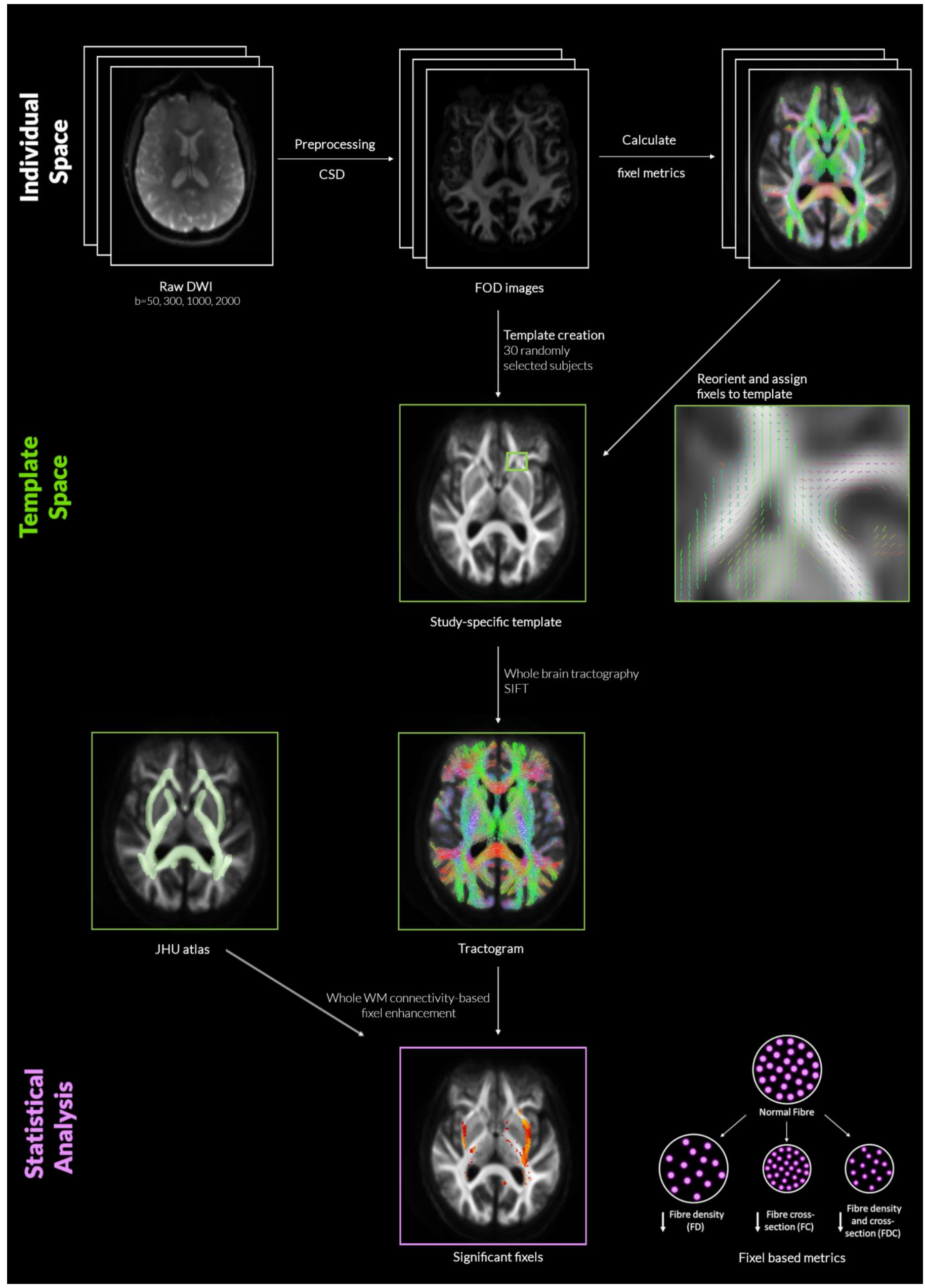




\section{Results}

Demographics and results of clinical assessments are seen in Table 1. PD high and PD low visual performers were well matched in terms of baseline cognitive performance and MCI status, as well as disease duration, motor severity and levodopa equivalent dosage. MCI status was defined as those with MCI at baseline, and those who converted to MCI during follow-up. In total 26 patients had PD-MCI (13 at baseline and 13 converted) while the remaining 50 patients had normal cognition throughout.

\section{Longitudinal changes in cognition}

At baseline, visuospatial performance was lower, as expected, in PD low visual performers compared to PD high visual performers and controls (JLO: $\mathrm{H}=8.855$, $\mathrm{p}=0.012$ and Hooper: $\mathrm{H}=20.652$, $\mathrm{p}<0.001)$. In other cognitive domains, performance at individual cognitive tasks was not significantly different between the two groups, with the exception of lower performance in PD low visual performers on a test of executive function (Stroop interference: $\mathrm{H}=6.642, \mathrm{p}=0.036$ ) and on a memory task (Word recognition task: $\mathrm{H}=6.716, \mathrm{p}=0.035$ ). Visual performance at the two computer tasks was correlated with overall cognitive performance at baseline $(r=-0.306, p=0.011)$, as well as cognitive performance at follow up ( $\mathrm{r}=-0.386, \mathrm{p}=0.001)$ (Figures $2 \mathrm{~B}$ and $2 \mathrm{C}$ ).

Significant changes in longitudinal performance between PD low and PD high visual performers were seen only for MMSE ( $\mathrm{t}=-1.084, \mathrm{p}=0.024)$, and Letter fluency $(\mathrm{t}=-2.825, \mathrm{p}=0.025)$. However, overall cognitive performance was lower in PD low visual performers, who were more likely to develop MCI at longitudinal follow up (chi=7.031, p=0.008, Figure 2A).

\section{Figure 2. Longitudinal changes in cognition in patients with Parkinson's disease.}

A. Percentage of patients who developed mild cognitive impairment (PD-MCI) in PD low visual performers compared to PD high visual performers.

B. Correlation between visual performance at baseline and combined cognitive performance at 18 month follow up in patients with Parkinson's disease (95\% confidence interval). Visual performance is presented as the summed z score of the two computer-based visual tasks (Cats and Dogs task and Biological Motion task). Cognitive performance is presented as combined cognitive score (z scored against the performance of age-matched controls).

C. Longitudinal change in combined cognitive score, MMSE and MoCA in PD low visual performers, PD high visual performers and controls. Error bars represent $95 \%$ confidence intervals. MMSE: Mini-Mental State Examination, MoCA: Montreal cognitive assessment 

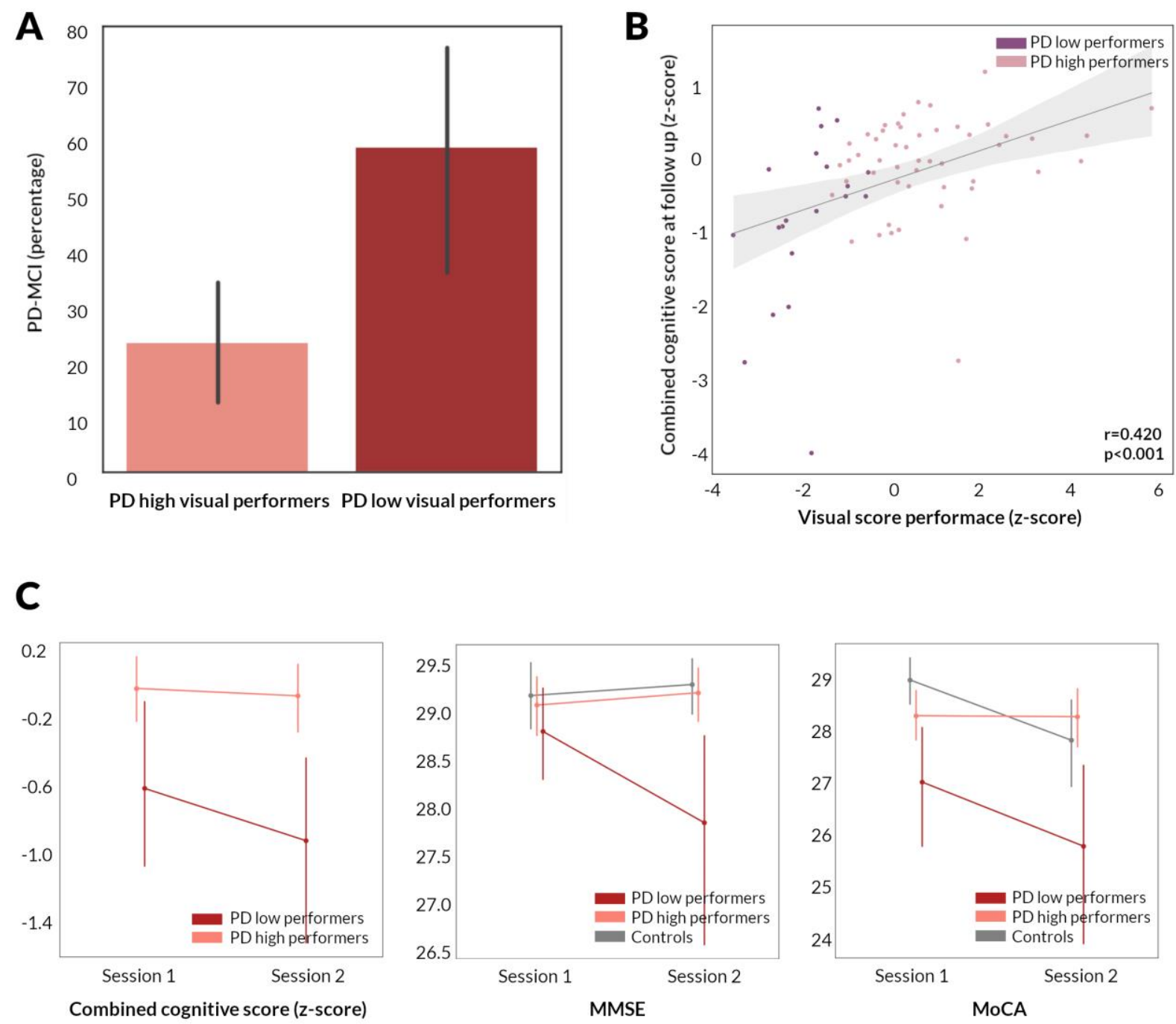

\section{Whole-brain longitudinal changes in white matter integrity}

No significant differences between patients with PD and normal controls was detected at whole brain fixel-based analysis or conventional voxel-based analysis, neither at baseline nor longitudinally.

1) Baseline changes in white matter micro-structure and macro-structure related to low visual performance in Parkinson's disease

PD low visual performers showed significant changes in white matter macro- and micro-structure compared to PD high visual performers, longitudinally (Figure 3) and already at baseline (Figure $4 \mathrm{~A}$ ), as we have shown previously ${ }^{13}$. At baseline, PD low visual performers showed reduction in FC within the splenium of the corpus callosum, the right cingulum and bilateral posterior thalamic radiation. Extensive micro-structural changes (reductions in FD) were also present at baseline in PD low visual performers, with reductions within the genu, body and splenium of the corpus callosum, 
the right internal capsule, the cingulum bilaterally, tapetum bilaterally, posterior thalamic radiations bilaterally, right hippocampus and the right corticospinal tract. Reductions were seen across the same regions in the combined FDC metric; these were particularly pronounced within the genu and splenium of the corpus callosum with greater than 30\% reductions in FDC. Figure 4A illustrates the extent of macro- and micro-structural changes seen in PD low visual performers at baseline.

2) White matter macro-structural changes in patients with Parkinson's disease and mild cognitive impairment

MCI status was less sensitive than visual performance in identifying baseline white matter alterations in patients with PD, with less tracts affected in PD-MCI. PD-MCI (including with normal cognition at baseline who would convert to PD-MCI) showed reductions in FC within the genu and splenium of the corpus callosum, the right posterior thalamic radiation, as well as the right hippocampus (Figure 4B). Whilst there were no statistically significant changes in FD, the combined FDC metric showed significant, over $30 \%$, reductions in the genu of the corpus callosum, and right hippocampus (Figure 4B). Given that white matter changes in PD-MCI and PD-low visual performers had differential spatial profiles direct comparison is difficult. However in both cases, the splenium of the corpus callosum was the most affected tract at baseline imaging. In direct comparison of mean FD, FC and FDC of the splenium, area under the curve was 0.615 for PD-MCI and 0.673 in PD-low visual performers, suggesting slightly higher sensitivity of visual performance for white matter alterations even within the same tract.

\section{Figure 3. Macrostructural white matter changes in patients with Parkinson's disease and low visual performance over time}

A. Changes in white matter macrostructure (as seen by reduction in fibre cross-section $(F C)$ ) in PD low visual performers compared to PD high visual performers (FWE-corrected $p<0.05$ ) at Session 1 (Baseline, left), at longitudinal change (difference between the two images, middle) and at Session 2 (18 months follow up, right). Results are presented as streamlines and coloured by percentage reduction.

B. Statistically significant ( FWE-corrected $p<0.05$ ) longitudinal reductions in fibre cross-section (FC) in PD low visual performers compared to PD high visual performers. Results are coloured by direction (anterior-posterior: green; superior-inferior: blue; left-right: red). 
bioRxiv preprint doi: https://doi.org/10.1101/2020 10.12 335547; this version posted October 12, 2020. The copyright holder for this preprint (which was not certified by peer review) is the author/funder, who has granted bioRxiv a license to display the preprint in perpetuity. It is made available under aCC-BY 4.0 International license.

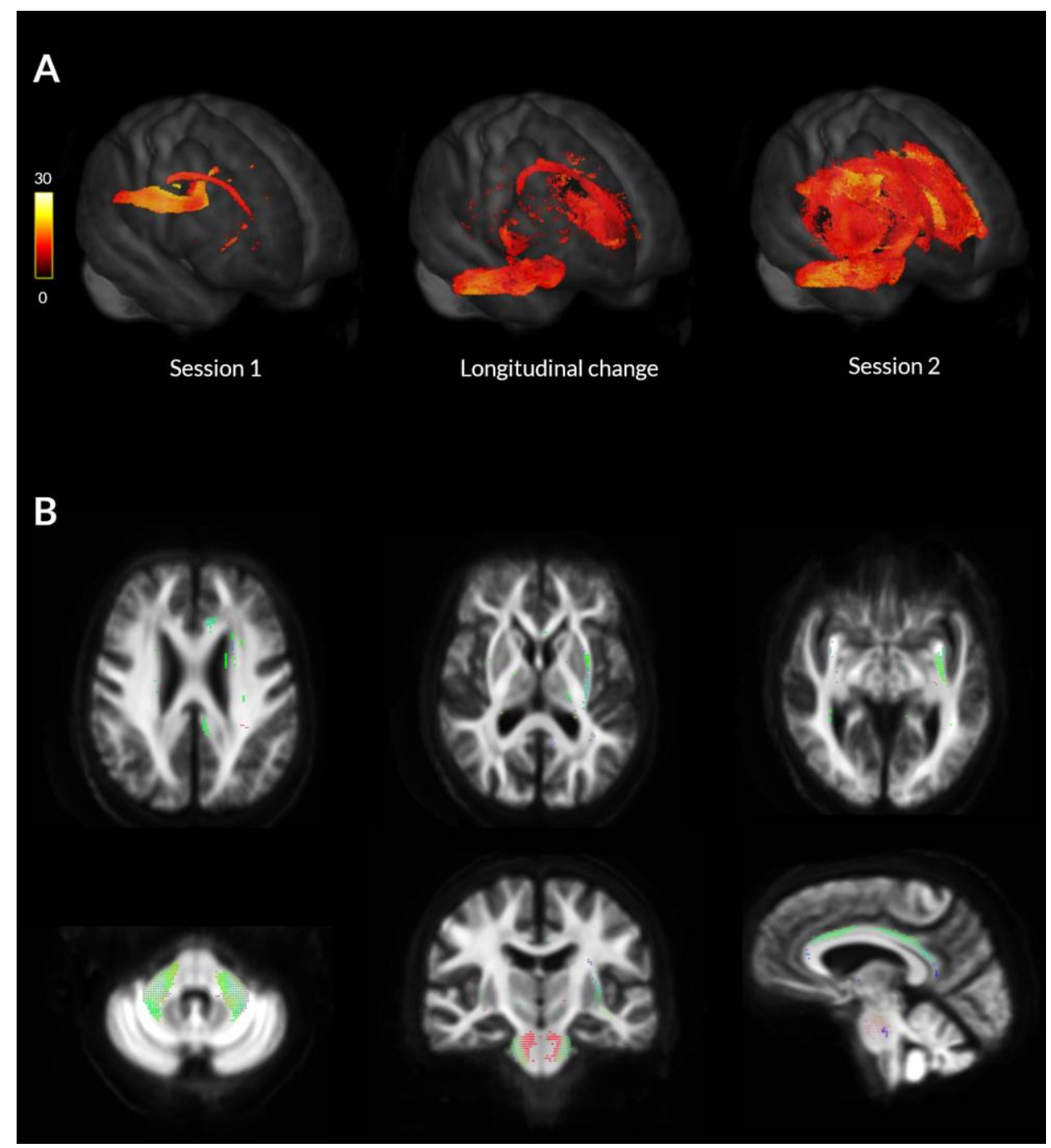




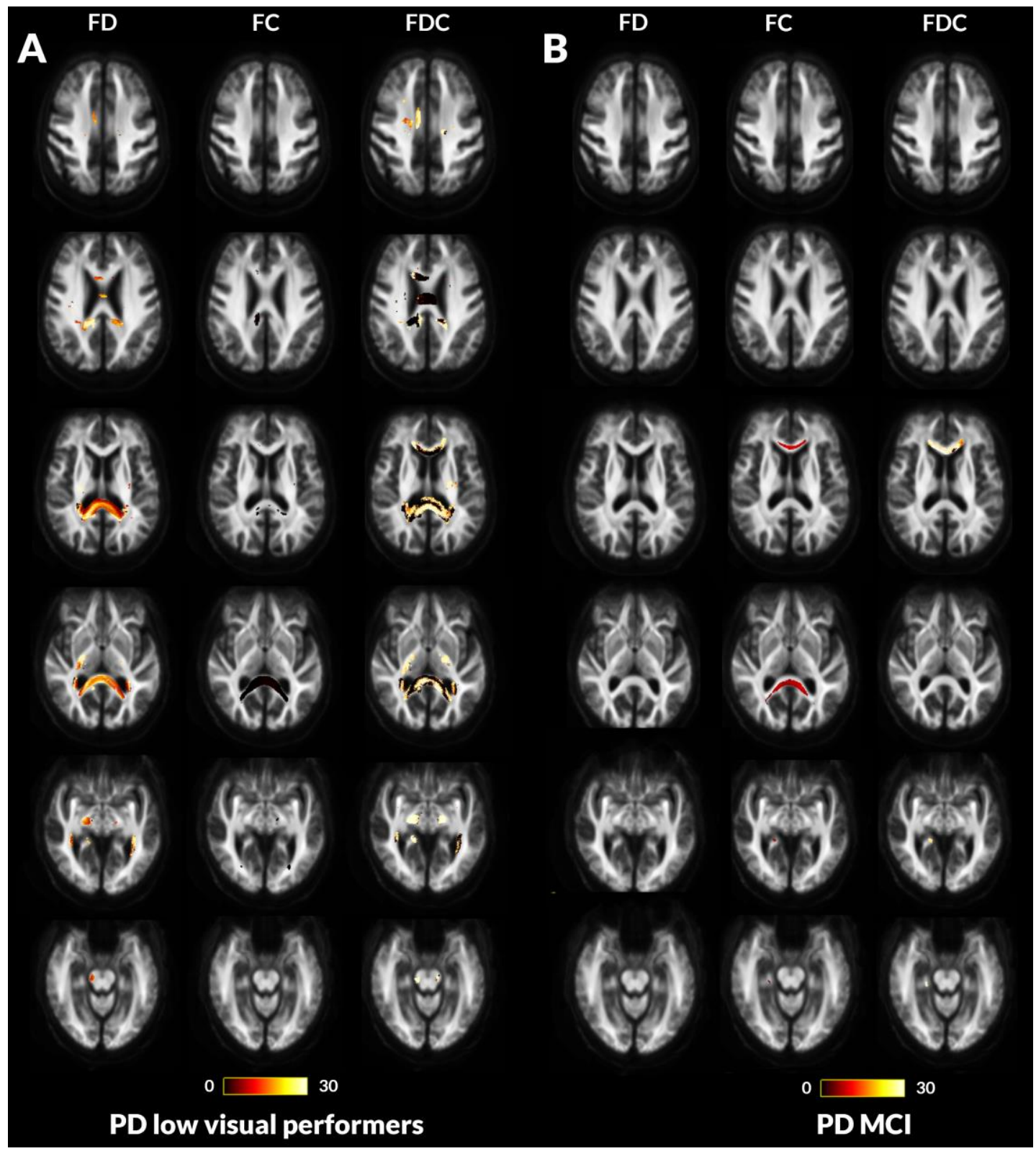


Figure 4. Fibre tract-specific reductions at baseline in PD low visual performers compared to PD high visual performers and PD with mild cognitive impairment compared to PD with normal cognition from whole-brain fixel-based analysis (opposite).

A. PD low visual performers showed widespread microstructural (changes in fibre density (FD)) with reductions within the genu, body and splenium of the corpus callosum, the right internal capsule, the cingulum bilaterally, tapetum bilaterally, posterior thalamic radiations bilaterally, right hippocampus and the right corticospinal tract. Macrostructural changes (changes in fibre density (FC) were also seen within the splenium of the corpus callosum, the right cingulum and bilateral posterior thalamic radiations. Changes in the combined FDC metric were seen within the genu, body and splenium of the corpus callosum, the right internal capsule, the cingulum bilaterally, tapetum bilaterally, posterior thalamic radiations bilaterally, right hippocampus and the right corticospinal tract; these represent impaired overall ability to relay information in these tracts in PD low visual performers.

B. Patients with Parkinson's disease who developed mild cognitive impairment (MCI) showed macrostructural (changes in fibre cross-section $(F C)$ ) within the genu and splenium of the corpus callosum, posterior thalamic radiations bilaterally and the right hippocampus.

Changes in the combined FDC metric are seen in the genu, and the right hippocampus; this represents impaired overall ability to relay information in these tracts in PD-MCI compared to PD with normal cognition (NC). No changes were seen in the FD metric for this patient group.

Results are displayed as streamlines; these correspond to fixels that significantly differed between PD low and high visual performers (FWE-corrected $p<0.05)$. Streamlines are coloured by percentage reduction (colourbars). 


\section{3) Longitudinal white matter alterations are seen in patients with Parkinson's disease and low} visual performance

PD low visual performers showed significant changes in white matter macro-structure, with up to $22 \%$ reductions in FC compared to PD high visual performers in whole brain fixel-based analysis. These macrostructural changes were extensive and involved multiple white matter tracts, including the middle cerebellar peduncles and pontine crossing parts bilaterally, the external capsules bilaterally, the left inferior and superior fronto-occipital fasciculi, the uncinate fasciculi bilaterally, the left cingulum, the left anterior and posterior corona radiata and the genu of the corpus callosum (Figure 3), and were corrected for age as well as gender and total intracranial volume. Importantly, cognitive measures, including MoCA scores and MCI conversion status (both assessing MCI at baseline and at follow up together and in separate sub-assessments within each group), as well as two clinical risk scores for dementia ${ }^{22,23}$ were not associated with longitudinal white matter changes at the micro- or at the macro-structural level.

\section{Voxel based analysis}

Conventional voxel-based analysis of FA and MD did not show any statistically significant differences after FWE correction for PD versus controls, or PD-MCI versus PD-NC at baseline or longitudinal follow up. PD low visual performers did not show any statistically significant differences at baseline. At longitudinal imaging, PD low visual performers showed reductions in FA within the left external capsule and right posterior thalamic radiation and increase in MD within the splenium of the corpus callosum.

\section{Tract of interest analysis}

Widespread reductions were seen in mean FC in PD low visual performers compared to PD high visual performers in tract of interest analysis. Specifically, at baseline after correction for age, gender and total intracranial volume, significant changes were seen across the corpus callosum (genu: $t=-$ 0.086, FDR-corrected $\mathrm{q}=0.002$, body: $\mathrm{t}=-0.076, \mathrm{q}=0.002$, splenium: $\mathrm{t}=-0.099, \mathrm{q}=0.002)$, the left inferior fronto-occipital fasciculus $(\mathrm{t}=-0.037, \mathrm{q}=0.002)$ and the right superior longitudinal fasciculus $(\mathrm{t}=-0.027, \mathrm{q}=0.002)$ (Figure 5B). At longitudinal follow up, all 11 selected tracts showed significant reductions (FDR-corrected) in PD low visual performers compared to PD high visual performers (Figure 5C). Across patients with PD, higher reductions in mean FC across the 11 selected tracts were associated with worsening MoCA scores $(\mathrm{r}=0.258, \mathrm{p}=0.024)$ but not change in MMSE scores at 18 months follow up. 
bioRxiv preprint doi: https://doi.org/10.1101/2020.10.12.335547; this version posted October 12, 2020. The copyright holder for this preprint (which was not certified by peer review) is the author/funder, who has granted bioRxiv a license to display the preprint in perpetuity. It is made available under aCC-BY 4.0 International license.

A

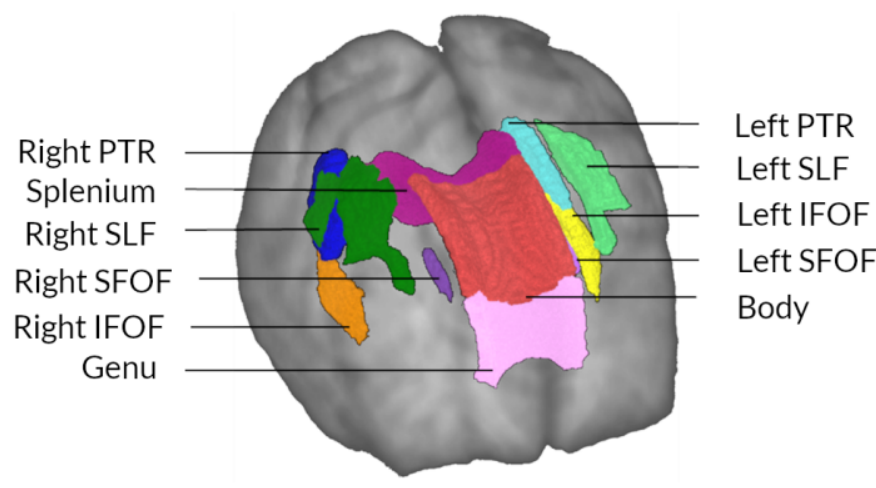

B

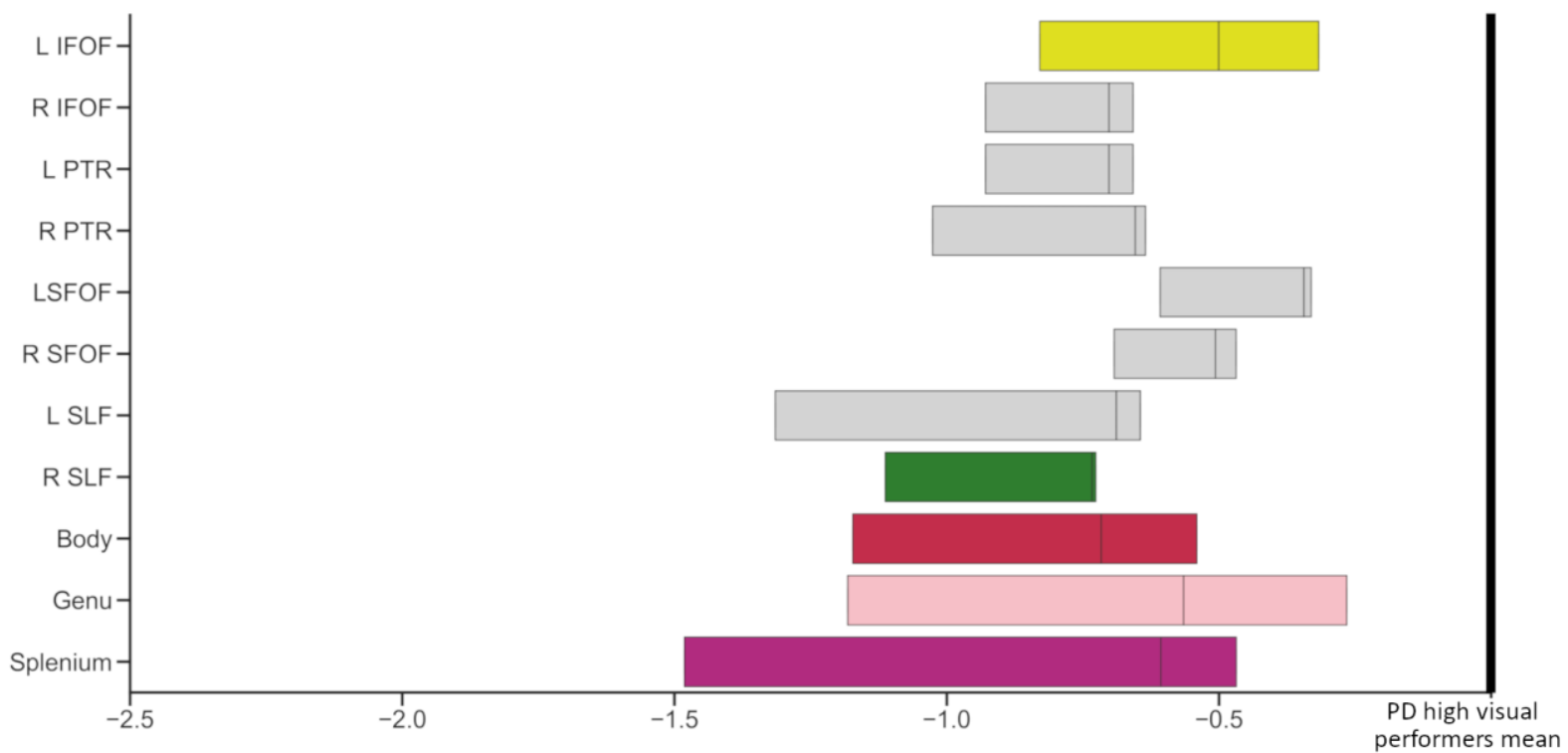

C

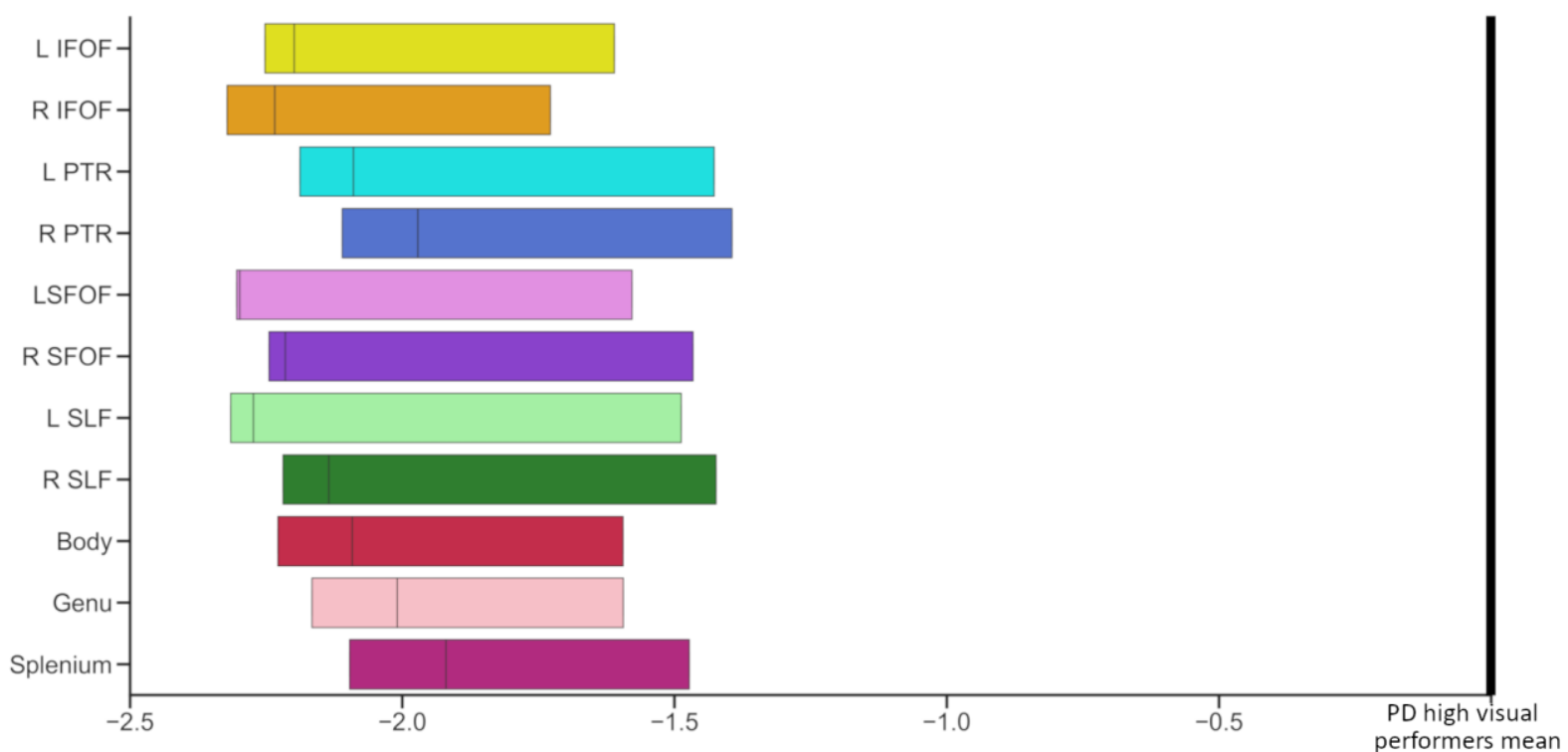


Figure 5. Significant tracts in Parkinson's low performers; tract of interest analysis (Opposite).

A. Anatomical representation of all analysed tracts. PTR: Posterior thalamic and optic radiations, SLF: Superior longitudinal fasciculi, IFOF: Inferior fronto-occipital fasciculi (segmentation includes the inferior longitudinal fasciculus), and SLF: Superior fronto-occipital fasciculi.

B. Baseline visit. Reduction (mean, 95\% CI) in fibre cross-section (FC) visualised as percentage reduction from the mean of patients with Parkinson's disease with high visual performance. Tracts with significantly reduced FC (FDR-corrected p-value <0.05) are shown in colour whilst tracts where there are no significant changes in FDC are plotted in grey. L: Left, R: Right.

C. Visit 2 (18 months follow up). Reduction (mean, 95\% CI) in fibre cross-section (FC) visualised as percentage reduction from the mean of patients with Parkinson's disease with high visual performance at follow-up. All 11 of the selected tracts showed significantly reduced FC (FDR corrected p-value <0.05). L: Left, R: Right.

\section{Discussion}

Low visual performance in Parkinson's disease was associated with cognitive decline and widespread white matter macrostructural changes at 18 months follow up. Specifically 1) low visual performance at baseline was correlated with worsening cognition and conversion to MCI at 18 months follow up; 2) baseline low visual performance was associated with further reductions in fibre cross-section at longitudinal follow up and 3) low performance on high-level visual tasks was associated with more extensive white matter changes at baseline than was seen in those patients who developed mild cognitive impairment on follow up. These findings provide evidence that visual changes in Parkinson's disease are a marker for incipient white matter degeneration and cognitive decline.

\section{White matter alterations precede cognitive impairment in Parkinson's disease}

We have previously described the white matter changes that are associated with low visual performance at baseline ${ }^{13}$. We found similar but significantly less extensive white matter changes in patients who later progressed to develop MCI, specifically involving the right hippocampus and the genu and splenium of the corpus callosum. Changes within the corpus callosum, particularly its most anterior and most posterior segments, have been previously reported in PD with cognitive impairment using diffusion tensor imaging ${ }^{10,25-28}$. Similar changes were seen using fixel-based 
analysis in association with disease severity, particularly non-motor symptoms, ${ }^{14}$ and in patients with PD and poor visual performance ${ }^{13}$.

The hippocampus has a higher Lewy body count and greater cholinergic deficit in PD dementia ${ }^{29}$ but studies assessing grey matter atrophy and neurotransmitter changes in vivo in PD with cognitive impairment have also demonstrated changes outside the hippocampus ${ }^{30-32}$, leading to debate regarding the relative importance of the hippocampus in PD dementia. In a prior meta-analysis using network-lesion mapping, our group has shown that hippocampal networks, particularly of the right hippocampus, which plays a crucial role in spatial memory ${ }^{33}$ are linked to PD dementia ${ }^{34}$. Our finding of right hippocampal tract involvement in PD-MCI provides corroborative evidence that hippocampal networks are implicated in the development of PD dementia.

In patients with low visual performance, in addition to more extensive alterations of both white matter micro-structure and morphology at baseline, additional macro-structural white matter changes developed at follow up. The right hippocampus was also affected in PD low visual performers together with its temporal lobe connections, such as the cingulum and inferior longitudinal fasciculus, which have also been implicated in Alzheimer's disease as well as Parkinson's with cognitive impairment ${ }^{35}$.

Long cortico-cortical tracts connecting frontal to occipital regions, such as the superior and inferior fronto-occipital fasciculi, and subcortical-cortical tracts, such as the external capsule, became bilaterally affected at follow up (particularly on the left hemisphere). The fronto-occipital fasciculi play a role in global cognition, attention, visual processing and executive function ${ }^{36,37}$. The external capsule acts as a route for cholinergic pathways and diffusion-derived metrics have been associated with cognitive performance in healthy older adults. ${ }^{38}$ Long-range connections play a crucial role in brain integration at network level ${ }^{39}$ and functional connectivity is reduced for fronto-occipital connections in PD with cognitive impairment. ${ }^{40,41}$

The cerebellar tracts, particularly the middle cerebellar peduncles showed significant macrostructural changes at follow up in PD low visual performers. The cortico-ponto-cerebellar pathway, which connects cortical regions to the cerebellum via the contralateral middle cerebellar peduncle, may play a key role in cognition ${ }^{42,43}$. In patients with Parkinson's disease, functional connectivity between the vermis and visual association and pre-frontal cortex is weaker than controls and correlates with cognitive performance, even in the absence of cerebellar volume loss or changes in cortical thickness ${ }^{21}$. Although traditionally regarded as unaffected by alpha-synuclein pathology and excluded from the Braak Lewy body pathological staging, recent evidence suggests that cerebellar 
nuclei and surrounding white matter do accumulate alpha-synuclein aggregates ${ }^{44}$. Our findings provide further support to structural white matter changes within the cerebellum in patients with PD and cognitive impairment.

In patients with PD and poor visual performance, we saw widespread white matter alterations, with interhemispheric connections in the corpus callosum, long fronto-occipital and hippocampal connections affected first, followed by diffuse macro-structural changes across multiple association tracts. These diffuse changes may explain the alterations in global functional and structural connectivity seen in Parkinson's disease ${ }^{45-47}$ and emphasise the new insights that network approaches can provide in our understanding of Parkinson's dementia.

\section{Impending cognitive decline and macrostructural white matter degeneration in patients with Parkinson's disease and visual dysfunction: an opportunity for intervention}

Baseline visual performance was associated with cognitive performance at follow-up in patients with PD; and poor visual performance predicted worsening cognition and the development of MCI. Prior work from our group has shown that visuo-perceptual deficits are linked to poorer cognitive performance at baseline and clinical scores for dementia ${ }^{5,16}$ as well as worsening MoCA at 1 year follow up ${ }^{17}$. This imaging study provides further evidence of impending cognitive impairment in patients with PD and visuo-perceptual deficits.

We also found that PD low visual performers exhibited significant and widespread reduction in fibre cross section, signalling widespread macro-structural white matter alterations at 18 months follow up. In contrast, patients with PD who are classified as high dementia risk by two clinically-derived scores $^{22,22}$ as well as those who developed MCI at follow up did not exhibit further white matter alterations, with PD-MCI showing established white matter degeneration at baseline. These findings raise the possibility that poor visual performance could represent a window of opportunity for therapeutic interventions in patients with $\mathrm{PD}$, by identifying patients with imminent but not yet clinically apparent cognitive impairment and white matter degeneration.

\section{Limitations and future directions}

Several considerations need to be taken into account when interpreting the results from our study. All raw imaging data in our cohort were visually inspected and clinically significant cerebrovascular disease was excluded, however, due to the acquired MRI sequences, we could not systematically quantify and control for white matter hyperintensities, which could lead to fibre density reduction ${ }^{48}$. This is in line with other published studies using fixel-based analysis, ${ }^{14,24,49,50}$ but future studies need 
to clarify the effect of white matter hyperintensities on fixel-based metrics. At both study timepoints, participants with PD underwent imaging on their usual dopaminergic medications. Corrected fractional anisotropy is not affected by levodopa ${ }^{51}$ therefore it is unlikely that dopaminergic medication would affect fixel-based metrics. In addition, levodopa equivalent doses were not significantly different between PD low and PD high visual performers at any study time-point. Follow up imaging and psychological testing was performed at 18 months; longer follow up times of patients with PD who will proceed to develop dementia could provide further insights to the temporal order of white matter degeneration in PD with cognitive impairment.

\section{Conclusions}

Poor visual function in Parkinson's disease is associated with worsening cognition and conversion to MCI at 18 months follow up. Poor visual performance was also associated with diffuse white matter macro-structural changes at follow up. These findings provide insights into the temporal pattern of white matter degeneration associated with cognitive impairment in Parkinson's disease and highlight an at-risk population for possible therapeutic intervention.

\section{Acknowledgements}

We gratefully acknowledge the support of NVIDIA Corporation with the donation of the Quadro P6000 GPU used for this research. The authors acknowledge the use of the UCL Myriad High Performance Computing Facility (Myriad@UCL), and associated support services, in the completion of this work. This research was also supported by the National Institute for Health Research University College London Hospitals Biomedical Research Centre.

\section{Author Contributions}

Study design and concept: AZ, RW, data collection: AZ, LAL, RW, imaging and statistical analysis: AZ, drafting and revision of the manuscript: AZ, PMC, LAL, AL, RW.

\section{Conflicts of Interest}

AZ is supported by an Alzheimer's Research UK Clinical Research Fellowship (2018B-001). PMC is supported by the National Institute for Health Research. RSW is supported by a Wellcome Clinical Research Career Development Fellowship (201567/Z/16/Z).

\section{References}


1. Williams-Gray CH, Mason SL, Evans JR, et al. The CamPaIGN study of Parkinson's disease: 10 -year outlook in an incident population-based cohort. J. Neurol. Neurosurg. Psychiatry 2013;84(11):1258-1264.

2. Hely MA, Reid WGJ, Adena MA, et al. The Sydney multicenter study of Parkinson's disease: the inevitability of dementia at 20 years. Mov. Disord. Off. J. Mov. Disord. Soc. 2008;23(6):837-844.

3. Anang JBM, Gagnon J-F, Bertrand J-A, et al. Predictors of dementia in Parkinson disease: a prospective cohort study. Neurology 2014;83(14):1253-1260.

4. Hamedani AG, Abraham DS, Maguire MG, Willis AW. Visual Impairment Is More Common in Parkinson's Disease and Is a Risk Factor for Poor Health Outcomes. Mov. Disord. Off. J. Mov. Disord. Soc. 2020;

5. Leyland L-A, Bremner FD, Mahmood R, et al. Visual tests predict dementia risk in Parkinson disease. Neurol. Clin. Pract. 2019;10.1212/CPJ.0000000000000719.

6. Toledo JB, Gopal P, Raible K, et al. Pathological $\alpha$-synuclein distribution in subjects with coincident Alzheimer's and Lewy body pathology. Acta Neuropathol. (Berl.) 2016;131(3):393-409.

7. Chung CY, Koprich JB, Siddiqi H, Isacson O. Dynamic changes in presynaptic and axonal transport proteins combined with striatal neuroinflammation precede dopaminergic neuronal loss in a rat model of AAV alpha-synucleinopathy. J. Neurosci. Off. J. Soc. Neurosci. 2009;29(11):33653373.

8. Volpicelli-Daley LA, Luk KC, Patel TP, et al. Exogenous $\alpha$-Synuclein Fibrils Induce Lewy Body Pathology Leading to Synaptic Dysfunction and Neuron Death. Neuron 2011;72(1):57-71.

9. Agosta F, Canu E, Stefanova E, et al. Mild cognitive impairment in Parkinson's disease is associated with a distributed pattern of brain white matter damage. Hum. Brain Mapp. 2014;35(5):1921-1929.

10. Kamagata K, Zalesky A, Hatano T, et al. Gray Matter Abnormalities in Idiopathic Parkinson's Disease: Evaluation by Diffusional Kurtosis Imaging and Neurite Orientation Dispersion and Density Imaging. Hum. Brain Mapp. 2017;

11. Tournier J-D, Mori S, Leemans A. Diffusion tensor imaging and beyond. Magn. Reson. Med. 2011;65(6):1532-1556.

12. Raffelt DA, Tournier J-D, Smith RE, et al. Investigating white matter fibre density and morphology using fixel-based analysis. NeuroImage 2017;144(Pt A):58-73.

13. Zarkali A, McColgan P, Leyland L-A, et al. Fiber-specific white matter reductions in Parkinson hallucinations and visual dysfunction. Neurology 2020;

14. Rau Y-A, Wang S-M, Tournier J-D, et al. A longitudinal fixel-based analysis of white matter alterations in patients with Parkinson's disease [Internet]. NeuroImage Clin. 2019;24[cited 2020 Jul 17 ] Available from: https://www.ncbi.nlm.nih.gov/pmc/articles/PMC6889638/

15. Kaul S, Elble RJ. Impaired pentagon drawing is an early predictor of cognitive decline in Parkinson's disease. Mov. Disord. Off. J. Mov. Disord. Soc. 2014;29(3):427-428. 
16. Weil RS, Pappa K, Schade RN, et al. The Cats-and-Dogs test: A tool to identify visuoperceptual deficits in Parkinson's disease. Mov. Disord. 2017;32(12):1789-1790.

17. Weil RS, Winston JS, Leyland L-A, et al. Neural correlates of early cognitive dysfunction in Parkinson's disease [Internet]. Ann. Clin. Transl. Neurol. 2019;0(0)[cited 2019 May 13 ] Available from: https://onlinelibrary.wiley.com/doi/abs/10.1002/acn3.767

18. Weil RS, Schwarzkopf DS, Bahrami B, et al. Assessing cognitive dysfunction in Parkinson's disease: An online tool to detect visuo-perceptual deficits. Mov. Disord. 2018;33(4):544-553.

19. Emre M, Aarsland D, Brown R, et al. Clinical diagnostic criteria for dementia associated with Parkinson's disease. Mov. Disord. Off. J. Mov. Disord. Soc. 2007;22(12):1689-1707; quiz 1837.

20. Litvan I, Goldman JG, Tröster AI, et al. Diagnostic Criteria for Mild Cognitive Impairment in Parkinson's Disease: Movement Disorder Society Task Force Guidelines. Mov. Disord. Off. J. Mov. Disord. Soc. 2012;27(3):349-356.

21. Maiti B, Koller JM, Snyder AZ, et al. Cognitive correlates of cerebellar resting-state functional connectivity in Parkinson disease. Neurology 2020;94(4):e384-e396.

22. Liu G, Locascio JJ, Corvol J-C, et al. Prediction of cognition in Parkinson's disease with a clinical-genetic score: a longitudinal analysis of nine cohorts. Lancet Neurol. 2017;16(8):620-629.

23. Schrag A, Siddiqui UF, Anastasiou Z, et al. Clinical variables and biomarkers in prediction of cognitive impairment in patients with newly diagnosed Parkinson's disease: a cohort study. Lancet Neurol. 2017;16(1):66-75.

24. Mito R, Raffelt D, Dhollander T, et al. Fibre-specific white matter reductions in Alzheimer's disease and mild cognitive impairment. Brain 2018;141(3):888-902.

25. Bledsoe IO, Stebbins GT, Merkitch D, Goldman JG. White matter abnormalities in the corpus callosum with cognitive impairment in Parkinson disease. Neurology 2018;91(24):e2244e2255.

26. Deng B, Zhang Y, Wang L, et al. Diffusion tensor imaging reveals white matter changes associated with cognitive status in patients with Parkinson's disease. Am. J. Alzheimers Dis. Other Demen. 2013;28(2):154-164.

27. Hanganu A, Houde J-C, Fonov VS, et al. White matter degeneration profile in the cognitive cortico-subcortical tracts in Parkinson's disease. Mov. Disord. Off. J. Mov. Disord. Soc. 2018;33(7):1139-1150.

28. Melzer TR, Watts R, MacAskill MR, et al. White matter microstructure deteriorates across cognitive stages in Parkinson disease. Neurology 2013;80(20):1841-1849.

29. Hall H, Reyes S, Landeck N, et al. Hippocampal Lewy pathology and cholinergic dysfunction are associated with dementia in Parkinson's disease. Brain J. Neurol. 2014;137(Pt 9):2493-2508.

30. Klein A, Ghosh SS, Bao FS, et al. Mindboggling morphometry of human brains. PLOS Comput. Biol. 2017;13(2):e1005350. 
31. Melzer TR, Watts R, MacAskill MR, et al. Grey matter atrophy in cognitively impaired Parkinson's disease. J. Neurol. Neurosurg. Psychiatry 2012;83(2):188-194.

32. Burton EJ, McKeith IG, Burn DJ, et al. Cerebral atrophy in Parkinson's disease with and without dementia: a comparison with Alzheimer's disease, dementia with Lewy bodies and controls. Brain 2004;127(4):791-800.

33. Ezzati A, Katz MJ, Zammit AR, et al. Differential association of left and right hippocampal volumes with verbal episodic and spatial memory in older adults. Neuropsychologia 2016;93(Pt B):380-385.

34. Weil RS, Hsu JK, Darby RR, et al. Neuroimaging in Parkinson's disease dementia: connecting the dots [Internet]. Brain Commun. 2019;1(1)[cited 2019 Nov 1 ] Available from: https://academic.oup.com/braincomms/article/1/1/fcz006/5529671

35. Bubb EJ, Metzler-Baddeley C, Aggleton JP. The cingulum bundle: Anatomy, function, and dysfunction. Neurosci. Biobehav. Rev. 2018;92:104-127.

36. Hau J, Sarubbo S, Perchey G, et al. Cortical Terminations of the Inferior Fronto-Occipital and Uncinate Fasciculi: Anatomical Stem-Based Virtual Dissection [Internet]. Front. Neuroanat.

2016;10[cited 2019 May 14 ] Available from:

https://www.ncbi.nlm.nih.gov/pmc/articles/PMC4877506/

37. Wu Y, Sun D, Wang Y, Wang Y. Subcomponents and Connectivity of the Inferior FrontoOccipital Fasciculus Revealed by Diffusion Spectrum Imaging Fiber Tracking. Front. Neuroanat. 2016;10:88.

38. Nolze-Charron G, Dufort-Rouleau R, Houde J-C, et al. Tractography of the external capsule and cognition: A diffusion MRI study of cholinergic fibers. Exp. Gerontol. 2020;130:110792.

39. van den Heuvel MP, Kahn RS, Goñi J, Sporns O. High-cost, high-capacity backbone for global brain communication. Proc. Natl. Acad. Sci. U. S. A. 2012;109(28):11372-11377.

40. Abós A, Baggio HC, Segura B, et al. Discriminating cognitive status in Parkinson's disease through functional connectomics and machine learning. Sci. Rep. 2017;7:45347.

41. Kawabata K, Watanabe H, Hara K, et al. Distinct manifestation of cognitive deficits associate with different resting-state network disruptions in non-demented patients with Parkinson's disease. J. Neurol. 2018;265(3):688-700.

42. Palesi F, De Rinaldis A, Castellazzi G, et al. Contralateral cortico-ponto-cerebellar pathways reconstruction in humans in vivo: implications for reciprocal cerebro-cerebellar structural connectivity in motor and non-motor areas [Internet]. Sci. Rep. 2017;7[cited 2020 Jul 21 ] Available from: https://www.ncbi.nlm.nih.gov/pmc/articles/PMC5634467/

43. Salmi J, Pallesen KJ, Neuvonen T, et al. Cognitive and motor loops of the human cerebrocerebellar system. J. Cogn. Neurosci. 2010;22(11):2663-2676.

44. Seidel K, Bouzrou M, Heidemann N, et al. Involvement of the cerebellum in Parkinson disease and dementia with Lewy bodies. Ann. Neurol. 2017;81(6):898-903.

45. Colon-Perez LM, Tanner JJ, Couret M, et al. Cognition and connectomes in nondementia idiopathic Parkinson's disease. Netw. Neurosci. Camb. Mass 2018;2(1):106-124. 
46. Galantucci S, Agosta F, Stefanova E, et al. Structural Brain Connectome and Cognitive Impairment in Parkinson Disease. Radiology 2017;283(2):515-525.

47. Lopes R, Delmaire C, Defebvre L, et al. Cognitive phenotypes in parkinson's disease differ in terms of brain-network organization and connectivity. Hum. Brain Mapp. 2017;38(3):1604-1621.

48. Dhollander T, Raffelt D, Connelly A. Towards interpretation of 3- tissue constrained spherical deconvolution results in pathology. [Internet]. Honolulu, Hawai: 2017 p. 1815.[cited 2019 May 14 ] Available from: https://www.researchgate.net/publication/307863133_Unsupervised_3tissue_response_function_estimation_from_single-shell_or_multishell_diffusion_MR_data_without_a_co-registered_T1_image

49. Grazioplene RG, Bearden CE, Subotnik KL, et al. Connectivity-enhanced diffusion analysis reveals white matter density disruptions in first episode and chronic schizophrenia. NeuroImage Clin. 2018;18:608-616.

50. $\mathrm{Mu}$ J, Chen T, Li P, et al. Altered white matter microstructure mediates the relationship between hemoglobin levels and cognitive control deficits in end-stage renal disease patients. Hum. Brain Mapp. 2018;39(12):4766-4775.

51. Chung JW, Burciu RG, Ofori E, et al. Parkinson's disease diffusion MRI is not affected by acute antiparkinsonian medication. NeuroImage Clin. 2017;14:417-421. 
bioRxiv preprint doi: https://doi org/10.1101/2020.10.12.335547; this version posted October 12,2020 . The copyright holder for this preprint (which was not certified by peer review) is the author/funder, who has granted bioRxiv a license to display the preprint in perpetuity. It is made available under aCC-BY 4.0 International license.

Table 1. Demographics and results of clinical assessments

\begin{tabular}{|c|c|c|c|c|}
\hline Characteristic & $\begin{array}{c}\text { Controls } \\
n=25\end{array}$ & $\begin{array}{c}\text { PD high } \\
\text { visual } \\
\text { performers } \\
\text { n= 54 } \\
\end{array}$ & $\begin{array}{c}\text { PD low visual } \\
\text { performers } \\
n=22\end{array}$ & Statistic \\
\hline Age (years) & $67.4(8.2)$ & $63.7(7.5)$ & $69.3(7.6)$ & $\begin{array}{r}F=7.119 \\
p=0.001^{a, b}\end{array}$ \\
\hline Male $(\%)$ & $12(48)$ & $26(48.1)$ & $15(68.2)$ & $\begin{array}{c}\mathrm{x}^{2}=2.890 \\
\mathrm{p}=0.089\end{array}$ \\
\hline Years of education & $17.8(2.2)$ & $16.9(2.9)$ & $18.1(2.5)$ & $\begin{array}{l}\mathrm{F}=1.919 \\
\mathrm{p}=0.152\end{array}$ \\
\hline \multicolumn{5}{|l|}{ Vision } \\
\hline Contrast sensitivity (Pelli Robson) * & $1.8(0.2)$ & $1.8(0.2)$ & $1.7(0.1)$ & $\begin{array}{l}\mathrm{H}=17.377 \\
\mathrm{p}=0.001^{\mathrm{a}, \mathrm{c}}\end{array}$ \\
\hline Acuity (LogMar) * & $-0.1(0.3)$ & $-0.1(0.2)$ & $-0.1(0.1)$ & $\begin{array}{l}\mathrm{H}=3.232 \\
\mathrm{p}=0.199\end{array}$ \\
\hline Colour vision (D15) & $1.3(2.4)$ & $2.7(8.9)$ & $3.0(4.9)$ & $\begin{array}{l}\mathrm{H}=1.934 \\
\mathrm{p}=0.380\end{array}$ \\
\hline \multicolumn{5}{|l|}{ General cognition } \\
\hline MCI & - & $13(24.1)$ & $13(59.1)$ & $\begin{array}{c}\mathrm{x}^{2}=2.933 \\
\mathrm{p}=0.087\end{array}$ \\
\hline MOCA & $29.0(1.1)$ & $28.3(1.9)$ & $27.0(2.7)$ & $\begin{array}{l}\mathrm{H}=8.333 \\
\mathrm{p}=0.016^{\mathrm{c}}\end{array}$ \\
\hline MMSE & $29.2(0.9)$ & $29.1(1.7)$ & $28.8(1.2)$ & $\begin{array}{l}H=1.534 \\
p=0.464\end{array}$ \\
\hline \multicolumn{5}{|l|}{ Mood } \\
\hline HADS anxiety & $3.5(3.4)$ & $5.4(3.7)$ & $6.5(3.9)$ & $\begin{array}{c}\mathrm{H}=8.201 \\
\mathrm{p}=0.017^{\mathrm{b}, \mathrm{c}}\end{array}$ \\
\hline HADS depression & $1.2(1.5)$ & $3.2(2.8)$ & $5.7(3.4)$ & $\begin{array}{l}H=28.898 \\
p<0.001^{\text {a,b }},\end{array}$ \\
\hline \multicolumn{5}{|l|}{ Disease specific measures } \\
\hline Years from diagnosis & - & $3.8(2.3)$ & $4.9(2.8)$ & $\begin{array}{l}\mathrm{t}=-1.787 \\
\mathrm{p}=0.078\end{array}$ \\
\hline UPDRS total score & - & $42.7(16.3)$ & $53.7(28.0)$ & $\begin{array}{l}t=-2.102 \\
p=0.039\end{array}$ \\
\hline UPDRS motor score & - & $22.4(10.2)$ & $25.8(16.2)$ & $\begin{array}{l}\mathrm{t}=-1.094 \\
\mathrm{p}=0.278\end{array}$ \\
\hline LEDD & - & 401.4 (211.6) & $492.8(225.5)$ & $\begin{array}{c}\mathrm{t}=-1.653 \\
\mathrm{p}=0.102\end{array}$ \\
\hline RBDSQ & - & $4.0(2.3)$ & $4.5(1.9)$ & $\begin{array}{l}t=-0.839 \\
p=0.404\end{array}$ \\
\hline
\end{tabular}

All data shown are mean $(S D)$ except gender and $M C I$.

Statistical comparisons shown are across the 3 groups with subscript for post-hoc results, except for disease specific measures (comparing PD low and high visual performers). In bold characteristics that significantly differed between groups.

a Statistically significant difference between PD high visual performers and PD low visual performers, ${ }^{\mathrm{b}}$

Statistically significant difference between PD high visual performers and controls, ${ }^{\mathrm{c}}$ Statistically significant difference between PD low visual performers and controls

* Best binocular score used; LogMAR: lower score implies better performance, Pelli Robson: higher score implies betterr performance.

${ }^{+}$Higher values imply worse image quality, - Higher values imply better image quality

MCI: mild cognitive impairment; HADS: Hospital anxiety and depression scale; MMSE: Mini-mental state examination; MOCA: Montreal cognitive assessment; UPDRS: Unified Parkinson's Disease Rating Scale, LEDD: Levodopa Equivalent Dose; RBDSQ: REM sleep behaviour disorder scale. 